\title{
DESCRIPTION D'UNE FRAYÈRE ET COMPORTEMENT DE REPRODUCTION DE LA GRANDE ALOSE (ALOSA ALOSA L.) DANS LE COURS SUPÉRIEUR DE LA LOIRE
}

\author{
P. BOISNEAU, Catherine MENNESSON-BOISNEAU, \\ et J.L. BAGLINIĖRE \\ Laboratoire d'Ecologie Hydrobiologique \\ Station de Physiologie et d'Ecologie des Poissons - INRA \\ 65, rue de St-Brieuc - 35042 RENNES CEDEX
}

\section{RÉSUMÉ}

Une frayère de grande Alose (Alosa alosa L.) a été décrite dans le cours supérieur de la Loire et le comportement de reproduction des adultes a été observé durant toute la période de frai en 1988. Le site était caractérisé par la présence d'un épi et par l'abondance de matériaux fins dans le substrat (sables et graviers). La reproduction était essentiellement nocturne avec un pic d'intensité très net au milieu de la nuit. L'activité de frai a été reliée négativement à la valeur journalière du débit d'eau et ne semble pas être influencée par la température de l'eau.

Le nombre de géniteurs a été estimé à environ 34.200 individus.

Les résultats montrent que : (1) cette frayère devait être un site de reproduction forcée et non typique de la grande Alose, et (2) apparaissait comme une des plus importantes observées sur les grands fleuves français.

Mots-clés : Grande Alose, Loire, reproduction, comportement.

\section{DESCRIPTION OF A SPAWNING GROUND \\ AND REPRODUCTIVE BEHAVIOUR OF THE ALLIS SHAD (ALOSA ALOSA L.) IN THE UPPER R. LOIRE}

\section{ABSTRACT}

A spawning ground of Allis Shad (Alosa alosa L.) was described in the upper R. Loire and the reproductive behaviour was observed during the course of spawning season in 1988 . The spawning ground was characterized by the presence of a groyne and by the abundance of fine materials in the substrate (sand an gravel). Reproduction was mainly nocturnal with a stronger intensity at mid-night. The spawning activity was correlated negatively with the daily flow rate and was not influenced by water temperature. The number of adults was estimated to about 34.200 individuals.

Results showed that this spawning ground could be a campelled spawning ground, not typical for Allis Shad, and was one of the most important observed in large French streams.

Key-words : Allis Shad, Loire, reproduction, behaviour.

\section{INTRODUCTION}

Une étude sur les populations de grande Alose (Alosa alosa L.) a débuté sur le bassin de la Loire en 1984. Dans un premier temps, elle a porté essentiellement sur l'activité de migration (BOISNEAU et al., 1985) et sur les caractéristiques biologiques des adultes (MENNESSONBOISNEAU et al., 1986). 
A l'exception des travaux de ROULE (1923) et LE CLERC (1941), il n'existait jusqu'à présent aucune description de site de frai et de comportement de reproduction de la grande Alose en Loire. Par contre, quelques observations récentes ont été faites dans les rivières du Sud-Ouest de la France : Garonne (CASSOU-LEINS, CASSOU-LEINS, 1981) Adour (BOIGONTIER, 1987). De même, pour une espèce d'Alose particulièrement étudiée en Amérique du Nord, Alosa sapidissima, très proche d'A. alosa, très peu de travaux ont été réalisés dans ce domaine (CHITTENDEN, 1976). Ainsi dans un deuxième temps, l'étude des populations de grande Alose en Loire s'est poursuivie en 1988 par une phase de description d'une frayère, localisée dans la partie supérieure du bassin, et du comportement de reproduction. La finalité de cette étude était double : (1) connaître les caractéristiques et le fonctionnement d'une frayère actuelle de grande Alose sur la Loire ; (2) apporter, dans ce domaine, des observations complémentaires à celles, trop peu nombreuses, réalisées en France sur d'autres systèmes fluviaux.

\section{TECHNIQUES ET MÉTHODES}

La Loire a une longueur de $1.012 \mathrm{~km}$ pour une superficie de bassin versant de 115.000 $\mathrm{km}^{2}$. Son débit moyen annuel à Montjean est de $860 \mathrm{~m}^{3} / \mathrm{sec}$ (ANONYME, 1986). La zone d'étude se situe en aval du barrage de Decize-St-Léger-des-Vignes (576 km de l'océan) qui constitue le point de blocage majeur des migrations anadromes sur le cours supérieur de la Loire (BAGLINIÈRE et al., 1988) (Figure 1).

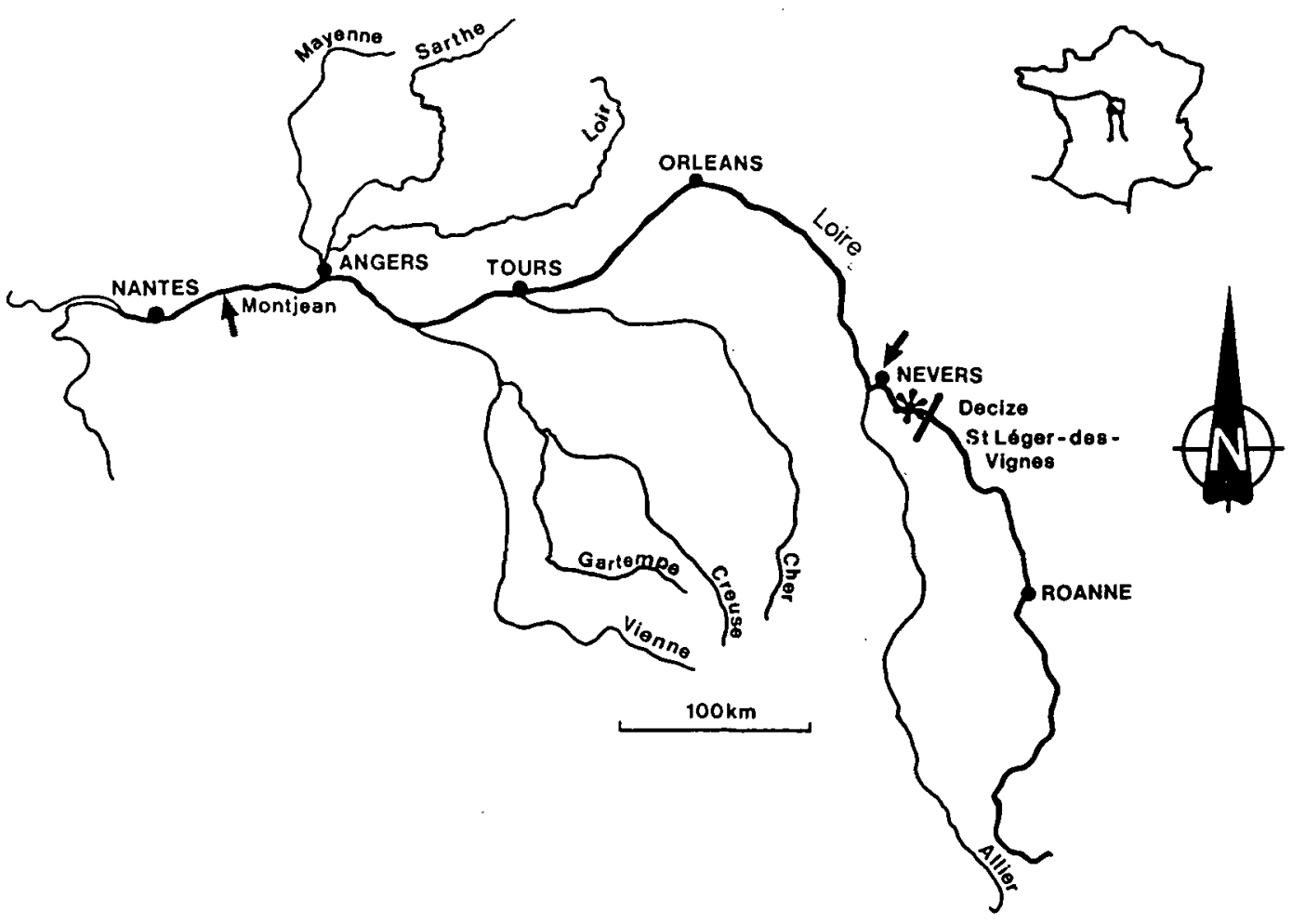

Figure 1 : Localisation du site d'étude de la frayère de grande Alose de St-Léger-desVignes sur la Loire en 1988

/Barrage

$\checkmark$ Localisation des enregistrements des débits

Figure 1 : Shad spawning ground at St-Léger-des-Vignes in the R. Loire in 1988 Dam Enregistred water flow 
Cette étude a comporté deux volets :

\section{Description de la zone de frayère}

La morphologie de la zone de frayère a été décrite le 28 juin 1988 pour un débit de $65 \mathrm{~m}^{3} /$ $\mathrm{sec}$. Sur des transects effectués en bateau, la profondeur de l'eau, la vitesse du courant et la nature du substrat sont relevées tous les 10 mètres. Les classes de granulométrie retenues sont celles mentionnées par Champigneulle (1978) :

\begin{tabular}{lll} 
Types de Substrat & $:$ & Classes granulométriques \\
\hline vaseux & $:$ & $<0,02 \mathrm{~mm}$ \\
sableux & $:$ & de $0,02 \mathrm{~mm}$ à $2 \mathrm{~mm}$ \\
gravier & $:$ & de $2 \mathrm{~mm}$ à $2 \mathrm{~cm}$ \\
galet & $:$ & de $2 \mathrm{~cm}$ à $10 \mathrm{~cm}$ \\
cailloux & $:$ de $10 \mathrm{~cm}$ à $20 \mathrm{~cm}$ \\
bloc & $:$ de $20 \mathrm{~cm}$ à $50 \mathrm{~cm}$ \\
roc & $:>50 \mathrm{~cm}$
\end{tabular}

\section{Déroulement de la reproduction}

Les observations sont effectuées du 2 mai au 27 juillet 1988, de nuit, en lumière naturelle (lune) ou à l'aide de lampes torches. Deux types d'informations sont notées :

1) l'activité de frai est quantifiée en comptabilisant le nombre de "bulls" qui correspondent à la manifestation comportementale de la ponte des aloses (CASSOU-LEINS et CASSOULEINS, 1981). Le comptage se fait à la vue et/ou à l'ouie, les observateurs étant situés au sommet de la berge, à l'amont de la zone de frayère en début de période ( $A$, figure 2 ), se déplaçant progressivement vers l'épi $(B)$ pour terminer à son extrémité $(C)$ en fin de période. Seul, le nombre de bulls sûrs est pris en compte dans les résultats.

2) des observations comportementales précises sont réalisées par des observateurs placés à l'extrémité de l'épi ( $C$, figure 2), sur les phénomènes de pontes se déroulant dans un rayon de moins de cinq mètres. La durée des bulis, leur sens de rotation et le nombre de couples y participant sont notés. Un suivi continu est effectué durant trente-deux jours sur la période du 17 mai au 26 juillet. Deux journées sont interrompues, l'une pour prospection à l'aval (30-31 juin), l'autre pour cause d'orage (4-5 juillet).

La température de l'eau est prise au début de chaque observation journalière et est mesurée en continu sur le site du barrage. Les valeurs de débits utilisées dans cette étude sont celles enregistrées à Nevers sur la Loire (Figure1).

Le rapport des sexes et l'état de maturité sont contrôlés sur des géniteurs capturés au coul et au carrelet au pied du barrage et sur la zone de frayère du 3 mai au 27 juillet. 

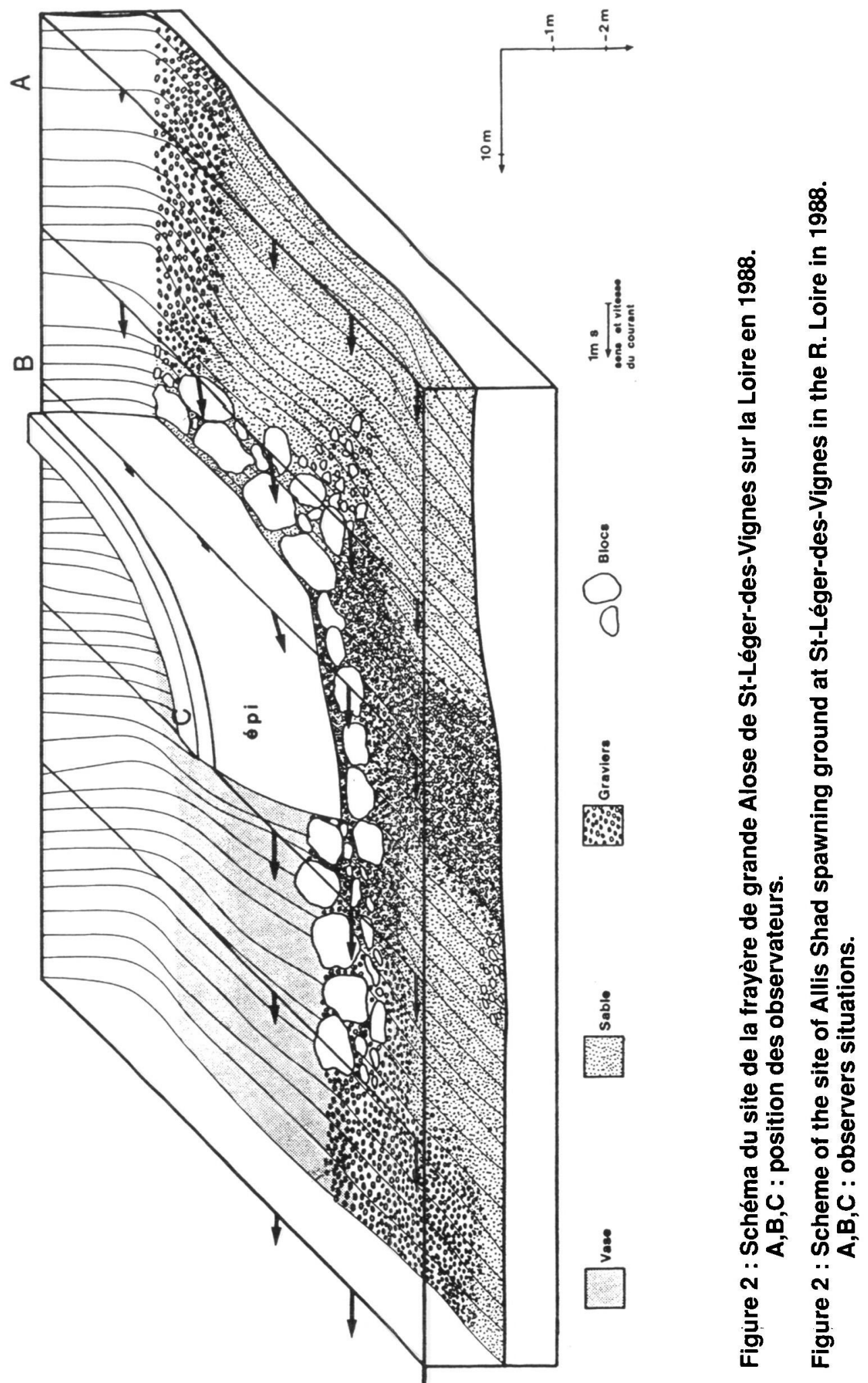


\section{RÉSULTATS}

Description de la zone de frayère (Figure 2)

Lazone de frayère se trouve à environ $2 \mathrm{~km}$ à l'aval du barrage sur la rive droite, à proximité de la station d'épuration de St-Léger-des-Vignes. Le site se distingue par la présence d'un épi de $35 \mathrm{~m}$ de long, disposé en arc de cercle, dont l'extrémité est orientée trois quarts aval.

La zone de reproduction est située plus précisément $200 \mathrm{~m}$ à l'amont de cet épi jusqu'à $50 \mathrm{~m}$ à l'aval lorsque le débit décroît. Les substrats dominants sont constitués de sable puis de gravier, les galets étant absents. La profondeur augmente de l'amont vers l'aval (de 0,95 m à $1,60 \mathrm{~m}$ ) et du large vers la rive droite (de $0,90 \mathrm{~m}$ à 2,06 m). La vitesse du courant est de 0,45 $\mathrm{m} / \mathrm{sec}$ en bordure et s'élève progressivement vers l'épi pour être maximale au large de son extrémité $(0,90 \mathrm{~m} / \mathrm{sec})$.

\section{Déroulement de la reproduction}

\section{Période et activité générale}

Aucune activité de frai n'est constatée avant le 16 mai. Par contre, le 24 mai, on assiste à une reproduction déjà importante (768 bulls) (Fig. 3 ).

L'activité de frai est très irrégulière avec un minimum de 82 bulls le 17 juin et un maximun de 2.587 bulls le 27 juin. La reproduction semble avoir pris fin le 27 juillet puisqu'il n'est plus compté que 60 bulls la nuit précédente (Figure 3), et que les géniteurs contrôlés ont totalement expulsé leurs produits sexuels.

Durant la période de reproduction, la température de l'eau fluctue entre $12{ }^{\circ} \mathrm{C}$ et $25^{\circ} \mathrm{C}$ mais en s'élevant progressivement. Le débit de la Loire varie entre 50 et $850 \mathrm{~m}^{3} / \mathrm{sec}$ mais en

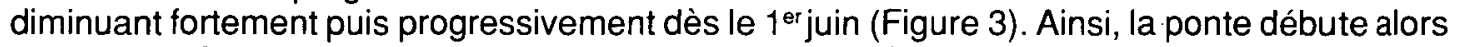
que la température de l'eau est de $16^{\circ} \mathrm{C}$ et le débit très élevé.

Aucune relation linéaire n'est trouvée entre d'une part le nombre de bulls et d'autre part les valeurs de la température de l'eau, ses variations et celles du débit. Par contre, une relation négative significative $(p<0,05)$ est établie entre le nombre de bulls $(B)$ et les valeurs du débit moyen journalier $(D)$ :

$$
B=-1,8035 D+1378,26(r=-0,3854 ; n=32) \text { : }
$$
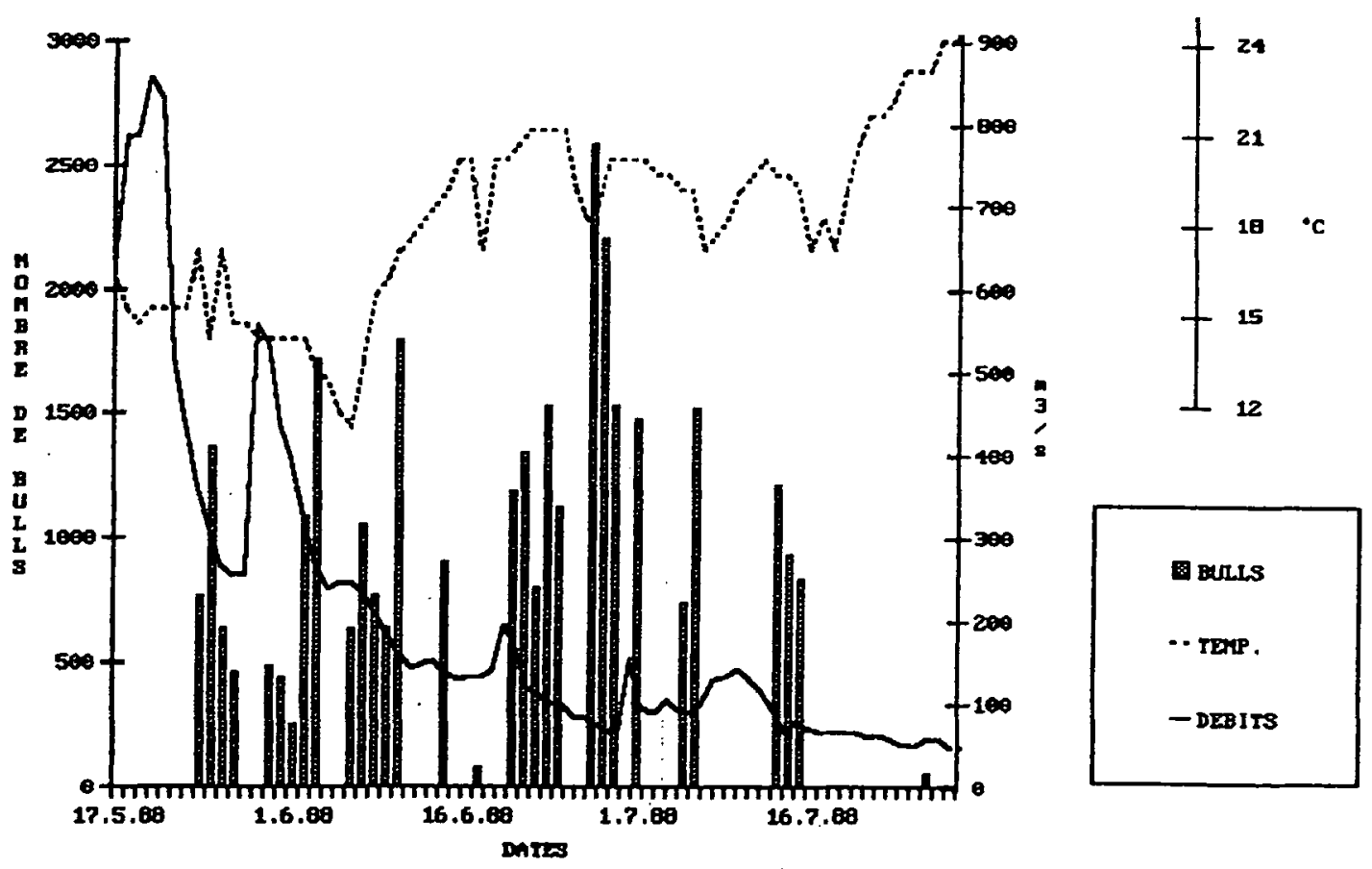

Figure 3 : Activité de frai (nombre de bulls), débits et température de l'eau sur la frayère de grande Alose de St-Léger-des-Vignes sur la Loire en 1988.

Figure 3 : Spawning activity (number of «bulls»), rivers flow and water temperature on the Allis Shad spawning ground at St-Léger-des-Vignes in the R. Loire in 1988. 
L'activité de reproduction a lieu presque exclusivement de nuit. Elle débute vers 21 h 30 pour progresser lentement jusqu'à $22 \mathrm{~h} 30$. Puis le nombre de bulls augmente rapidement pour culminer à 2 h 00 du matin (en moyenne 76,3 pour 15 minutes) et ensuite décroître aussi vite jusqu'à 5 h 00 (Figure 4). Ainsi, la distribution du nombre moyen de bulls par 15 minutes au cours d'une nuit suit parfaitement une loi normale (vérification par construction de la droite de Henry) (SCHWARTZ, 1986). Le rapport des sexes moyen contrôlé par captures de 324 géniteurs durant toute la période de reproduction est de 1,25 mâle pour une femelle.

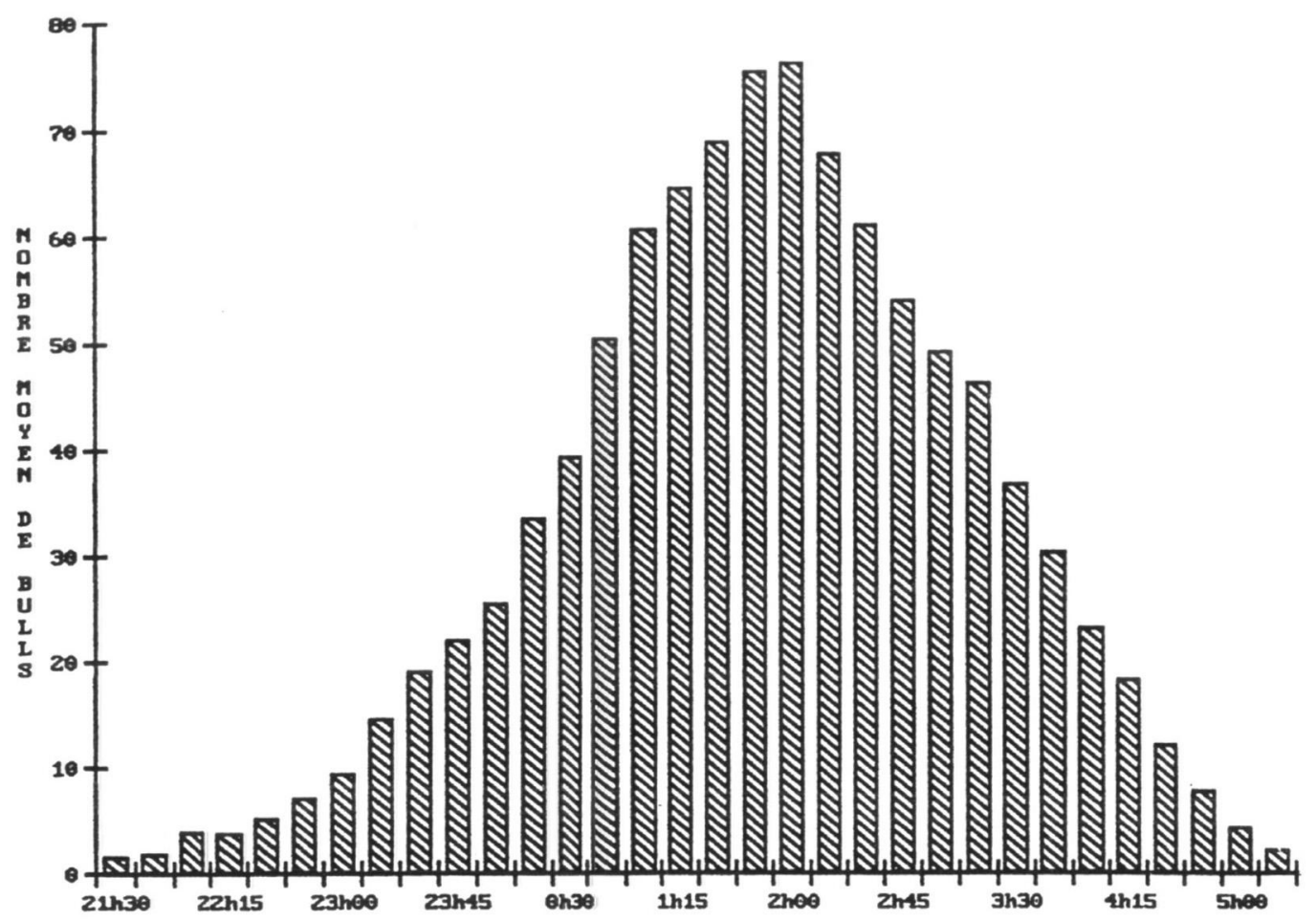

Figure 4 : Variation de l'activité de frai (nombre moyen de bulls) de grande Alose durant un cycle nocturne sur la frayère de St-Léger-des-Vignes en 1988.

Figure 4 : Variation of Allis Shad spawning activity (mean number of «bulls») during a nycthemeral phase on the spawning ground at St-Léger-des-Vignes in 1988.

\section{Comportement de ponte}

Au crépuscule, les aloses montent à la surface de l'eau pour venir se rassembler sur le site de frai (présence de multiples sillages). Puis, la nuit tombée, l'activité de frai commence. Les couples se tiennent alors à l'interface eau-air, flanc contre flanc.

Ils frappent violemment la surface de l'eau de la nageoire caudale en décrivant des cercles d'environ 1 à $1,20 \mathrm{~m}$ de diamètre. Cette activité dure de 1,93 à 10,29 secondes pour une moyenne de 4,7 ( 222 observations). Pendant ce temps, les produits génitaux sont expulsés et la fécondation a lieu dans le tourbillon provoqué.

Un bull peut donner lieu à plusieurs pontes. En effet, sur 573 bulls étudiés, il a pu être observé la ponte de 1, 2, 3 (ou plus) couples dans les proportions suivantes : 1 couple $78,18 \%$; 2 couples $21,12 \% ; 3$ couples ou plus $0,70 \%$. Ainsi, une relation entre le nombre de ponte $(P)$ et de bulls (B) est établie : $P=1,2252 B$.

Sur $44 \%$ des bulls étudiés, les géniteurs ont tourné dans le sens des aiguilles d'une montre ; $51,8 \%$ ont tourné dans le sens inverse ; $0,7 \%$ des géniteurs ont inversé le sens de rotation au cours du bull. Enfin, $3,5 \%$ de ces bulls ont été provoqués par deux couples tournant. chacun dans leur sens. 


\section{DISCUSSION}

Les résultats de cette étude permettent de préciser trois aspects de la phase de reproduction de la grande Alose en Loire.

\section{Caractéristiques de la zone de frayère}

Une frayère de grande Alose est généralement caractérisée par un "profond" à l'amont et une zone peu profonde à courant rapide à l'aval (CASSOU-LEINS et CASSOU-LEINS, 1981). Dans cette dernière zone, le substrat est constitué de cailloux ou de galets (diamètre $>7 \mathrm{~cm}$ ) et le courant doit avoir une vitesse supérieure à $1 \mathrm{~m} / \mathrm{sec}$, être turbulent et provoquer des remous (ROULE, 1923 ; LE CLERC, 1941 ; CASSOU-LEINS et CASSOU-LEINS, 1981, CASSOULEINS et CASSOU-LEINS, 1986). Enfin, pour CASSOU-LEINS et CASSOU-LEINS (1981, $1985,1990)$ la caractéristique dominante d'une frayère d'Alose serait plutôt la courantologie et la granulométrie, cette dernière conditionnant la survie des œufs et des larves.

La frayère de St-Léger-des-Vignes ne remplit pas toutes ces conditions, notamment absence de galets. Le choix de ce site pourrait s'expliquer, avant tout, par la présence de l'épi qui provoque localement une accélaration du courant. De plus, sur plusieurs kilomètres à l'aval de ce site, aucune zone remplissant les critères requis ne semble exister tant au niveau de la forme des courants que de la nature du substrat (dominance de sables et graviers). Par contre, des frayères typiques d'aloses ont existé sur les parties hautes de la Loire à l'amont de Roanne et sur l'Arroux (DUHAMEL DU MONCEAU, 1772 ; BENARDEAU, 1905 ; ROULE, 1923 ; LE CLERC, 1941 ; POITRINEAU, 1985). Or les observations vidéo réalisées à la même période montrent la grande diffuculté qu'ont les adultes d'aloses pour franchir le barrage de St-Légerdes-Vignes (BOISNEAU et al., 1989). Ainsi l'ensemble de ces considérations laissent penser que la frayère de Decize est un site de reproduction forcée de la grande Alose en Loire, pouvant affecter la réussite de ponte d'autant plus que ce site est proche de la station d'épuration de la ville de St-Léger-des-Vignes.

\section{Déroulement de la reproduction}

Certaines caractéristiques de la phase de reproduction sont en accord avec les quelques descriptions déjà réalisées au niveau national, notamment en ce qui concerne la période (mimai à fin juillet) le déroulement nocturne et la gamme de température de l'eau (18 à $24^{\circ} \mathrm{C}$ ) (ROULE, 1923 ; CASSOU-LEINS et CASSOU-LEINS, 1981, 1990). Cependant, dans l'étude en Loire, l'activité de frai a été observée à des températures de l'eau $\left(12^{\circ} \mathrm{C}\right)$ inférieures à la valeur du seuil $\left(14^{\circ} \mathrm{C}\right)$ mentionnée par ces mêmes auteurs. De plus, jusqu'à présent, aucune étude n'avait permis de relier l'activité de frai (nombre de bulls) au débit d'eau journalier. Cependant, la relation établie dans le cadre de l'étude a une portée restreinte dans la mesure où (1) elle est limitée à un site particulier, (2) elle nécessite une confirmation et un réajustement annuel, (3) elle surestime le nombre de bulls en fin de période de frai. Enfin, la comparaison de l'époque du pic

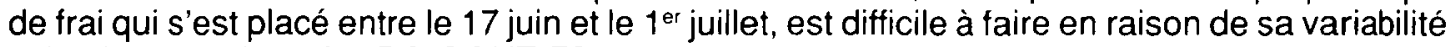
selon le site et l'année (BOIGONTIER, 1987).

Pour le rapport des sexes, CASSOU-LEINS et CASSOU-LEINS $(1985,1986$ et 1988) et BOIGONTIER (1987) le considèrent comme équilibré dans les populations du Sud-Ouest qu'ils ont étudiées alors que les observations de LE CLERC (1941) sur la Loire sont en accord avec les résultats présentés, à savoir un rapport des sexes en faveur des mâles.

Les observations effectuées sur la Loire quant aux phases comportementales prénuptiales et de reproduction proprement dite sont généralement en accord avec celles anciennes de ROULE (1923), de LE CLERC (1941), d'HOESTLANDT (1948) et plus récentes de CASSOULEINS et CASSOU-LEINS $(1981,1985,1990)$. Cependant, elles se distinguent de celles réalisées par ces derniers auteurs dans le Sud-Ouest par plusieurs aspects : (1) une proportion non négligeable de bulls donne lieu à la ponte de plusieurs couples ce qui signifie qu'en Loire un bull n'équivaut pas à une ponte ; (2) la rotation des couples ne se fait pas uniformément dans le même sens.

\section{Estimation du nombre de géniteurs}

L'estimation du nombre de géniteurs peut être réalisée à partir du nombre de bulls si l'on 
considère les critères utilisés par CASSOU-LEINS et CASSOU-LEINS (1985, 1986 et 1988) et BOIGONTIER (1987) à savoir

- le nombre de géniteurs sur la zone de frayère est fixe. II n'y a ni émigration ni immigration, — une femelle effectue 5 à 7 pontes sur le même site.

Cependant, toutes les nuits n'ayant pas fait l'objet d'un comptage pendant toute la période de reproduction, une estimation du nombre total de bulls est nécessaire. Celle-ci est faite en utilisant la relation linéaire débits-bulls pendant la période du 24 mai au 27 juillet, et la méthode des moyennes pondérées proposée par CASSOU-LEINS et CASSOU-LEINS (1985) et BOIGONTIER (1987) en dehors de cette période.

Ainsi, le nombre total de bulls est estimé à 72.343 pour la période complète de reproduction. Cette première estimation permet ensuite d'évaluer le nombre de pontes à 88.635 compte tenu de la liaison établie entre les deux paramètres dans cette étude. Enfin, en considérant le nombre de pontes par femelle et la valeur du rapport des sexes observés sur le site, le nombre de géniteurs est estimé entre 28.490 et 39.885 individus soit une moyenne numérique d'environ 34.200 et pondérale de 51,3 tonnes (poids moyen 1,5 kg). De même, sachant que le poids moyen des femelles sur le site était de $1,66 \mathrm{~kg}$ et considérant les valeurs de fécondité fournies par HOESTLANDT (1958, 125.000 ovocytes par kilogramme) et DAUTREY et LARTIGUE (1983, 156.000 ovocytes par kilogrammes), il est possible d'estimer la ponte des aloses entre 2,5 et 3,5 milliards d'œufs.

\section{CONCLUSION}

Cette étude de la description d'une zone frayère et du comportement de reproduction de la grande Alose en Loire confirme certaines observations réalisées sur d'autres systèmes fluviaux dans le cadre de travaux qui, au niveau national, restent très peu nombreux. Par ailleurs, elle apporte quelques éléments complémentaires de réflexion sur le comportement de reproduction pouvant avoir une incidence sur les méthodes actuellement utilisées pour le comptage des géniteurs. Ainsi, cela renforce la nécessité actuelle de mettre au point une méthode d'estimation fiable du nombre de généteurs d'aloses à partir d'une multiplication des observations dans l'espace et dans le temps sur un grand nombre de fleuves.

Cette étude fait également ressortir l'originalité et l'importance de la frayère de St-Légerdes-Vignes non seulement au niveau de la Loire elle-même (absence de frayères jusqu'au confluent avec l'Allier, frayères d'importance inconnue sur cette rivière) mais surtout au niveau des autres fleuves français. En effet, cette frayère apparaît comme très éloignée de la mer (plus de $500 \mathrm{~km}$ ) puisque les autres sont généralement situées à moins de $300 \mathrm{~km}$ de l'estuaire sur la Garonne (CASSOU-LEINS et CASSOU-LEINS, 1981, 1985), $100 \mathrm{~km}$ pour l'Adour (BOIGONTIER, 1987) ou $160 \mathrm{~km}$ pour la Dordogne (PUSTELINK, 1987). De plus, sur le plan quantitatif cette frayère s'avère comparable à celle de Lamagistère (CASSOU-LEINS et CASSOU-LEINS, 1988) et se placerait ainsi parmi les plus importantes actuellement répertoriées en France. Par contre, compte tenu de sa situation et de ses caractéristiques, cette frayère de St-Léger-des-Vignes apparaît comme un site forcé et non typique pour la reproduction de la grande Alose ce qui nécessitera dans les années à venir de suivre et de contrôler la réussite de ponte, celle-ci pouvant être affectée par une trop forte concentration de géniteurs et l'absence de galets.

\section{REMERCIEMENTS}

Cette étude a été réalisée dans le cadre d'un contrat avec le SRETIE et la Direction de la Protection de la Nature du Secrétariat d'Etat à l'Environnement. Le bon déroulement de ce travaił a pu être assuré grâce à la collaboration étroite et active de nombreux intervenants. Ainsi, nous remercions les gardes-pêche des départements de la Nièvre, de l'Allier, de la Saône-etLoire, de la Loire, du Cher et du Loiret ainsi que les responsables de leur Fédération. Nous remercions également tout le personnel des Délégations Régionales du Conseil Supérieur de la Pêche de Lyon, Dijon et de Clermont-Ferrand.

\section{BIBLIOGRAPHIE}

ANONYME, 1986. Répertoire des stations de jaugeage. Agence de Bassin Loire-Bretagne, $2 \mathrm{vol}$. 
BAGLINIĖRE J.L., C. BOISNEAU, P. BOISNEAU, 1988. Etude de la grande Alose (Alosa alosa) dans le bassin de la Loire; Rap. contrat SRETIE-INRA, Lab. Hydrobiol; $15 \mathrm{p}$.

BENARDEAU F., 1905. Pêche et reproduction du saumon en Loire. Berger-Levrault et Cie Ed., Paris-Nancy, 1 vol., $51 \mathrm{p}$.

BOIGONTIER B., 1987. Evaluation de l'impact du projet de seuil et de recalibrage de l'Adour (Toulouzette) sur les populations piscicoles et principalement sur les aloses. Rap. CEMAGREF Bordeaux, ALA, $80 \mathrm{p}$.

BOISNEAU P., BOISNEAU C., BAGLINIĖRE J.L., 1989. Migration et reproduction de la grande Alose (Alosa alosa L.) sur la Loire en 1988. Rap. contrat SRETIE-INRA, Lab. Ecol. Hydrobiol., $10 \mathrm{p}$.

BOISNEAU P., MENNESSON C., BAGLINIERE J.L., 1985. Observations sur l'activité de migration de la grande Alose, Alosa alosa L., en Loire (France). Hydrobiologia, 128, 277 284.

CASSOU-LEINS F., CASSOU-LEINS J.J., 1981. Recherches sur la biologie et l'halieutique des migrateurs de la Garonne et principalement de l'Alose, Alosa alosa L. Thèse $3^{\circ} \mathrm{cycle,} \mathrm{Inst.}$ Nat. Polytechn., Toulouse, $382 \mathrm{p}$.

CASSOU-LEINS F., CASSOU-LEINS J.J., 1985. Etude de la frayère d'aloses. Rap. ENSA Toulouse, $12 \mathrm{p}$.

CASSOU-LEINS F., CASSOU-LEINS J.J., 1986. Etude de la reproduction de l'Alose. Rap. ENSA Toulouse, $12 \mathrm{p}$.

CASSOU-LEINS F., CASSOU-LEINS J.J., 1988. Suivi de la réserve naturelle de la frayère d'alose. Année 1988. DEE, ENSA Toulouse, $12 \mathrm{p}$.

CASSOU-LEINS F., CASSOU-LEINS J.J., 1990. Réserve naturelle de la frayère d'aloses. Synthèse quinquennale. Rapport ENSA Toulouse, $57 \mathrm{p}$.

CHAMPIGNEULLE A., 1978. Caractéristiques de l'habitat piscicole et de la population de juvéniles sauvages de saumon atlantique (Salmo salarL.) sur le cours principal du Scorff (Morbihan). Thèse 3e cycle, Fac. Sci., Univ. Rennes, $92 \mathrm{p}$.

CHITTENDEN M.E., 1976. Present and historical spawning grounds and nurseries of American Shad, Alosa sapidissima, in the Delaware River. Fish Bull., 74 (2), 343-352.

DAUTREYR., LARTIGUEP., 1983. Recherches sur la migration des aloses (Alosa alosa) et des truites de mer (Salmo trutta) en Garonne (site de Golfech). Thèse $3^{\circ}$ cycle, Inst. Nat. Polytech. Toulouse, $212 \mathrm{p}$.

DUHAMEL DU MONCEAU M., 1772. Traité général des pesches et histoires des poissons qu'elles fournissent. Paris, Saillant et Nyon Ed., $2^{\circ}$ partie, section 3, 315-334.

HOESTLANDT H., 1948. Fécondation artificielle et incubation de l'alose du Rhône: Paralosa rhodoenensis Roule. Ann. Stat. Centr. Hydrobiol. Appl., 2, 223-228.

HOESTLANDT H., 1958. Reproduction de l'alose atlantique (Alosa alosa Linné) et transfert au Bassin Méditerranéen. Verh. Internat. Ver. Limnol., 13, 736-742.

LE CLERC M., 1941. Note sur les essais de multiplication artificielle de l'alose dans le bassin de la Loire. Bull. Fr. Piscic., 123, 27-37.

MENNESSON-BOISNEAU C., BOISNEAU P., BAGLINIĖRE J.L., 1986. Premières observations sur les caractéristiques biologiques des adultes de grande Alose (Alosa alosa L.) dans le cours moyen de la Loire. Acta Oecol. Oecol. Applic., 7, 337-353.

POITRINEAU A., 1985. La Loire, les peuples du fleuve. Horvath Ed., 151-163.

PUSTELNIK G., 1987. Les résultats de la campagne 1986 de piégeage et de capture dans la passe à poissons de Bergerac. Rap. Cons. Sup. Pêche, Dél. Rég. Midi-PyrénéesAquitaine, $10 \mathrm{p}$.

ROULE L., 1923. Notes sur les aloses de la Loire et de l'Aquitaine. Bull. Soc. Cent. Agric. Pêche, $30,14-22$.

SCHWARTZ D., 1986. Méthodes statistiques à l'usage des médecins et des biologistes. Flammarion Médecine Sciences, Ed., $318 \mathrm{p}$. 